\title{
BMJ Open Does glucagon-like peptide-1 (GLP-1) receptor agonist stimulation reduce alcohol intake in patients with alcohol dependence: study protocol of a randomised, double-blinded, placebo- controlled clinical trial
}

Kerstin K Antonsen, ${ }^{1}$ Mette K Klausen, ${ }^{1}$ Amanda S Brunchmann, ${ }^{1}$ Nina le Dous, ${ }^{1}$ Mathias E Jensen, ${ }^{1}$ Kamilla Woznica Miskowiak, ${ }^{1,2}$ Patrick M Fisher, ${ }^{3}$ Gerda K Thomsen, ${ }^{3}$ Henrik Rindom, ${ }^{4}$ Thomas P Fahmy, ${ }^{4}$ Sabine Vollstaedt-Klein, ${ }^{5}$ Helene Benveniste, ${ }^{6}$ Nora D Volkow, ${ }^{7}$ Ulrik Becker, ${ }^{8,9}$ Claus Ekstrøm, ${ }^{10}$ Gitte Moos Knudsen, ${ }^{3,11}$ Tina Vilsbøll, ${ }^{11,12}$ Anders Fink-Jensen ${ }^{1,11}$

To cite: Antonsen KK, Klausen MK, Brunchmann AS, et al. Does glucagonlike peptide-1 (GLP-1) receptor agonist stimulation reduce alcohol intake in patients with alcohol dependence: study protocol of a randomised, doubleblinded, placebo-controlled clinical trial. BMJ Open 2018;8:e019562. doi:10.1136/ bmjopen-2017-019562

- Prepublication history and additional material for this paper are available online. To view these files, please visit the journal online (http://dx.doi. org/10.1136/bmjopen-2017019562).

KKA and MKK contributed equally.

Received 14 November 2017 Revised 5 March 2018 Accepted 11 May 2018

Check for updates

For numbered affiliations see end of article.

Correspondence to

Professor Anders Fink-Jensen; Anders.Fink-Jensen@regionh.dk

\section{ABSTRACT}

Introduction Alcohol dependence is a major public health problem. It is underdiagnosed and undertreated. Even when treated, more than $2 / 3$ of patients in abstinenceoriented treatment will relapse within the first year. Thus, there is an urgent need for efficacious medical treatment of alcohol dependence. Glucagon-like peptide-1 (GLP1) receptor stimulation has proven to reduce alcohol consumption in preclinical experiments. However, the effect of GLP-1 receptor agonists in humans has to our knowledge, not yet been investigated.

Methods and analysis Design, participants and intervention: The effect of the once-weekly GLP-1receptor-agonist exenatide will be investigated in a double-blinded, placebo-controlled, randomised clinical trial. 114 outpatients will be recruited and randomised to treatment with either placebo or exenatide once weekly for 26 weeks as a supplement to cognitive-behavioural therapy. The primary endpoint is reduction in number of 'heavy drinking days'. The secondary endpoints include changes in total alcohol consumption, days without consumption, changes in brain activity and function, smoking status, cognition, measures of quality of life and changes in phosphatidylethanol as a biomarker of alcohol consumption from baseline to follow-up at week 26. Status: Currently recruiting patients.

Ethics and dissemination Ethical approval has been obtained. Before screening, all patients will be provided oral and written information about the trial. The study results will be disseminated by peer-review publications and conference presentations and has the potential to reveal a completely new medical treatment of alcohol dependence.

\section{INTRODUCTION}

Alcohol dependence is a major global public health problem across the world. ${ }^{1}{ }^{2}$ It is an underdiagnosed and undertreated ${ }^{3}$ condition
Strengths and limitations of this study

The trial is a double-blinded, randomised, placebo-controlled trial which is designed, to evaluate the effects of the glucagon-like peptide- 1 receptor agonist exenatide on alcohol consumption in patients with alcohol dependence.

- The study duration is 26 weeks which is longer than most previous studies investigating medical treatment of alcohol dependence.

- The biological basis for any demonstrated effect is investigated with brain imaging techniques and the biomarker phosphatidylethanol.

- A possible limitation is that the alcohol intake is self-reported which potentially could affect accuracy.

- Another limitation is that the study has no third treatment arm for comparing exenatide to one of the known compounds used in the clinic against alcohol use disorder, for example, disulfiram, acamprosate or naltrexone.

and more than 2/3 of patients in abstinence-oriented treatment will relapse within the first year. ${ }^{4}$ In Denmark, approximately $20 \%$ of the population is consuming more alcohol than recommended by the Danish National Board of Health. ${ }^{5}$ Further, $8.5 \%$ of the Danish adult population (16 years or older) has a 'risky', that is, potentially harmful, alcohol consumption, defined as more than 14 and 21 units of alcohol (one unit defined as $12 \mathrm{~g}$ of pure alcohol) for women and men per week, respectively. ${ }^{6}$ Three per cent fulfil the criteria for alcohol dependence. $^{7}$ 


\section{Psychological treatment}

One of the best documented treatments of alcohol dependence is cognitive-behavioural therapy (CBT) ${ }^{8}$ The underlying neuroanatomical basis of alcohol addiction and treatment effects of CBT are not yet established, although functional MRI (fMRI) studies have begun to elucidate the neural underpinnings of alcohol dependence. ${ }^{9}$ Alcohol-dependent patients have been found to display increased dorsal anterior cingulate cortex activation during spatial working memory (SWM), perhaps reflecting decreased prefrontal efficiency because of distracting alcohol-related thoughts. ${ }^{10}$ Interestingly, it was also recently demonstrated that alcohol-dependent patients also display increased neural activation to alcohol associated cues in mesocorticolimbic networks; which is normalised with psychological therapy. ${ }^{9}$

\section{Pharmacological treatment}

Pharmacological treatment of alcohol dependence is considered an important supplement to psychological therapy. ${ }^{11}$ Disulfiram, a substance that blocks alcohol-metabolising enzymes resulting in increased acetaldehyde concentrations, was introduced in Denmark in $1948 .^{12}$ Newer pharmacological agents, such as acamprosate, a gamma-aminobutyric acid receptor agonist and a N-Methyl-D-aspartate receptor antagonist; and naltrexone, a mu and kappa opioid receptor antagonist are now used as alternate treatments for alcohol dependence. However, these compounds have not gained widespread dissemination, probably because the effect of the substances is modest, with a less than $10 \%$ increase in abstinent rate compared with placebo. ${ }^{13}$ The antiepileptic compound topiramate has shown promising results in clinical trials ${ }^{14}$ and another pharmacological agent, nalmefene, with a mechanism of action somewhat similar to naltrexone, has very recently been approved by the European Medicines Agency as a medication for reducing alcohol consumption. ${ }^{15}$ Clearly, given the moderate success rates of $\mathrm{CBT}^{8}$ and the synergistic effects of adding pharmacological treatment - as described in this section - are quite limited, there is an urgent need for new and more efficient treatment modalities of alcohol dependence.

\section{Glucagon-like peptide-1 and GLP-1 receptor agonists}

Glucagon-like peptide-1 (GLP-1)-based therapy for the treatment of type 2 diabetes was introduced in $2006 .^{16}$ GLP-1 is an incretin hormone, which is secreted from endocrine $\mathrm{L}$ cells of the small intestine in response to nutrients in the gut lumen. ${ }^{17}$ GLP-1 conveys an insulinotropic effect through GLP-1 receptors (GLP-1R) on the beta cells of the pancreas and inhibits the secretion of glucagon from the alpha cells of the pancreas, which lower the blood glucose level. ${ }^{18}$ Naturally occurring GLP-1 is rapidly degraded within minutes by the enzyme, dipeptidyl peptidase-4. ${ }^{17}$ Exendin-4, originally isolated from the saliva of a lizard species, the Gila monster, has $53 \%$ sequence homology with human GLP-1 in its first 30 amino acids. Exenatide binds to the GLP-1R with high affinity $^{19}$ and acts as a receptor agonist, thus referred to as a GLP-1 receptor agonists (GLP-1RA).

\section{GLP-1RA: a potential new treatment for alcohol use disorder?}

GLP-1RA has a well-established effect on the food reward system which seems to be driven by two key mesolimbic brain regions, the ventral tegmental area (VTA) and nucleus accumbens (NAc). ${ }^{20}$ These regions are involved in the rewarding properties of food and to drugs of abuse, including alcohol. ${ }^{21-23}$ Interestingly, GLP-1 receptors (GLP-1R) are expressed in these brain reward regions (VTA and NAc), which are innervated by hindbrain GLP-1 neurons. ${ }^{21}$ A link between alcohol intake and GLP-1 has been demonstrated in studies and is considered to play a central role in the development of addiction to stimulant drugs (ie, cocaine, amphetamine, alcohol). ${ }^{24}$ The findings are consistent with the hypothesis, that systemic administration of GLP-1RA can influence the mesolimbic dopamine system and reward-seeking behaviours associated with alcohol dependence. ${ }^{25}$ Although the precise mechanism of action has not been elucidated in vivo, we recently reported that in vitro, exendin- 4 induces an upregulation of the dopamine transporter (DAT) function. ${ }^{26}$

Given this collective evidence, we aim to investigate whether the beneficial effect of the GLP-1RA, exenatide, on alcohol consumption in preclinical studies, can be translated to patients with known alcohol use disorder. ${ }^{27}$

\section{Hypothesis}

- Exenatide treatment will decrease alcohol consumption, measured as total number of heavy drinking days, in alcohol-dependent patients.

- Exenatide will induce upregulation of the striatal DAT availability, in alcohol-dependent patients.

- Exenatide will modulate neural responses in reward processing regions including NAc.

To test these hypothesis, we have designed a 26-week, clinical trial including 114 patients with known alcohol dependence. To explore the underlying neuromolecular mechanism(s) of the potential positive effect of exenatide versus placebo on alcohol consumption, we will obtain single-photon emission CT (SPECT) neuroimaging of DAT at weeks 0 and 26 in a subgroup of the patients. Further, the functional brain network modulated by the possible treatment effects will be investigated in a subgroup using fMRI at weeks 0 and 26 .

\section{METHODS AND ANALYSIS \\ Study design}

The present study is a 26-week, double-blinded, randomised, placebo-controlled clinical trial, designed to evaluate the effects of exenatide versus placebo in 114 patients, diagnosed with alcohol dependence according to the International Classification of Diseases, 10th Edition (ICD-10) and with an alcohol use disorder according to the The Diagnostic and Statistical Manual of Mental 
Disorders, Fifth Edition (DSM-5) criteria. The patients will be recruited from the Novavì outpatient clinics in Copenhagen, Denmark. To be eligible for participation, the patients will first undergo screening according to the inclusion and exclusion criteria. When consented and included, the patient will meet to get his/her weekly injection by the unblinded nurse. The nurse will collect a weekly alcohol diary and hand out a new one, for the following week.

\section{Participants and screening}

The patients will be recruited from outpatient units, specifically, the Novavi outpatient clinics in suburbs of Copenhagen, Denmark. All patients will receive psychosocial alcohol treatment based on psycho educative elements, motivational interviewing and CBT. Skilled staff members will be administering the psychosocial treatment in the clinic. The recruitment procedure starts as a prescreening when the patients contact the Novavi outpatient clinics, which are open outpatient clinics. All potentially eligible patients will be fully informed, verbally as well as in writing, of their rights and responsibilities while participating in the trial. Screening examinations will only be performed after the patient has agreed to participate and has signed the informed consent form.

At the time of screening, the patients will undergo a series of examinations to assure that all inclusion and none of the exclusion criteria are met. The patients will be asked general information about psychosocial factors, that is, education level, employment status and marital status. In addition, somatic symptoms and baseline medications will be registered. Blood samples and a urine tests will be collected for acute analysis according to the exclusion criteria. Furthermore, blood samples and urine samples will be saved for an investigational biobank that will allow more advanced analyses, for example, phosphatidylethanol (PEth). The most important tests and examinations are described in details in later sections. See also figure 1 for a complete schedule of events. The inclusion frequency will be regularly evaluated, and we have the option of including more trial centres to assure recruitment.

\section{Intervention}

The pharmacological intervention will be given as an add-on to the standardised psychosocial alcohol treatment paradigm. Exenatide is delivered from Region Hovedstadens Apotek as a powder with solvent for prolonged release injection (once weekly). Each singledose, dual-chamber pen contains $0.65 \mathrm{~mL}$ of diluent and $2 \mathrm{mg}$ of exenatide, which are isolated until mixed by the nurse administering the drug. The placebo will be supplied as prefilled saline syringes ( $0.9 \%$ saline), by Region Hovedstadens Apotek and will be administered in the same way and volume as exenatide. The unblinded nurse, with no involvement in the psychosocial treatment, will administer the drug injections, and the patients will be blindfolded while receiving the once-weekly injections. To promote participant retention, the nurse will contact the patient if he or she does not show up for the weekly injection.

\section{Inclusion criteria}

- Informed oral and written consent.

- Diagnosed with alcohol dependence according to the criteria of ICD-10, WHO and DSM-5 (for the equivalent diagnosis of alcohol use disorder).

- Alcohol Use Disorder Identification Test (AUDIT) score $>15 .{ }^{28}$

- Age 18-70years (both included).

- Heavy alcohol drinking defined as having alcohol consumption over $60 \mathrm{~g}$ of alcohol per day (men) or $48 \mathrm{~g}$ of alcohol per day (women) for at least 5 days in the past 30 days prior to inclusion measured by the Timeline Followback Method (TLFB) method.

\section{Exclusion criteria}

- Severe psychiatric disease, for example, a diagnosis of schizophrenia, paranoid psychosis, bipolar disorder or mental retardation.

- A history of delirium tremens or alcohol withdrawal seizures.

- No serious withdrawal symptoms at inclusion (defined as a score higher than 9 on Clinical Institute Withdrawal Assessment for Alcohol Scale, Revised at baseline examinations).

- Current or history of neurological disease including traumatic brain injury.

- Current or history of diagnosis of type 1 or type 2 diabetes or plasma haemoglobin A1c (HbA1c) $\geq 48$ $\mathrm{mmol} / \mathrm{mol}$ equivalent to $6.5 \%$ at inclusion.

- Women of childbearing potential who are pregnant, breast feeding or have intention of becoming pregnant within the next 9 months, or are not using contraceptives (during the whole study period) considered as highly effective. ${ }^{29}$

- Impaired hepatic function (liver transaminases $>3$ times upper normal limit).

- Impaired renal function (estimated glomerular filtration rate $(\mathrm{eGFR}))<50 \mathrm{~mL} / \mathrm{min})$.

- Impaired pancreatic function (any history of acute or chronic pancreatitis and/or amylase $>2$ times upper limit).

- S-triglycerides $>10 \mathrm{mmol} / \mathrm{L}$.

- History of medullary thyroid carcinoma (MTC) and/ or family history with MTC and/or multiple endocrine neoplasia syndrome type $2 .{ }^{30}$

- Cardiac problems defined as decompensated heart failure (New York Heart Association functional class III or IV), unstable angina pectoris and/or myocardial infarction within the last 12 months.

- Uncontrolled hypertension (systolic blood pressure $>180 \mathrm{~mm} \mathrm{Hg}$, diastolic blood pressure $>110 \mathrm{~mm} \mathrm{Hg}$ ).

- Concomitant pharmacotherapy against alcohol dependence, that is, disulfiram, naltrexone, acamprosate and nalmefene or treatment with any of these compounds within 1 month prior to inclusion. 
Study flow diagram

\section{Enrolment}

Day 1

Abstinence treatment, if needed

Supply of written patient information

and booking of an information meeting.

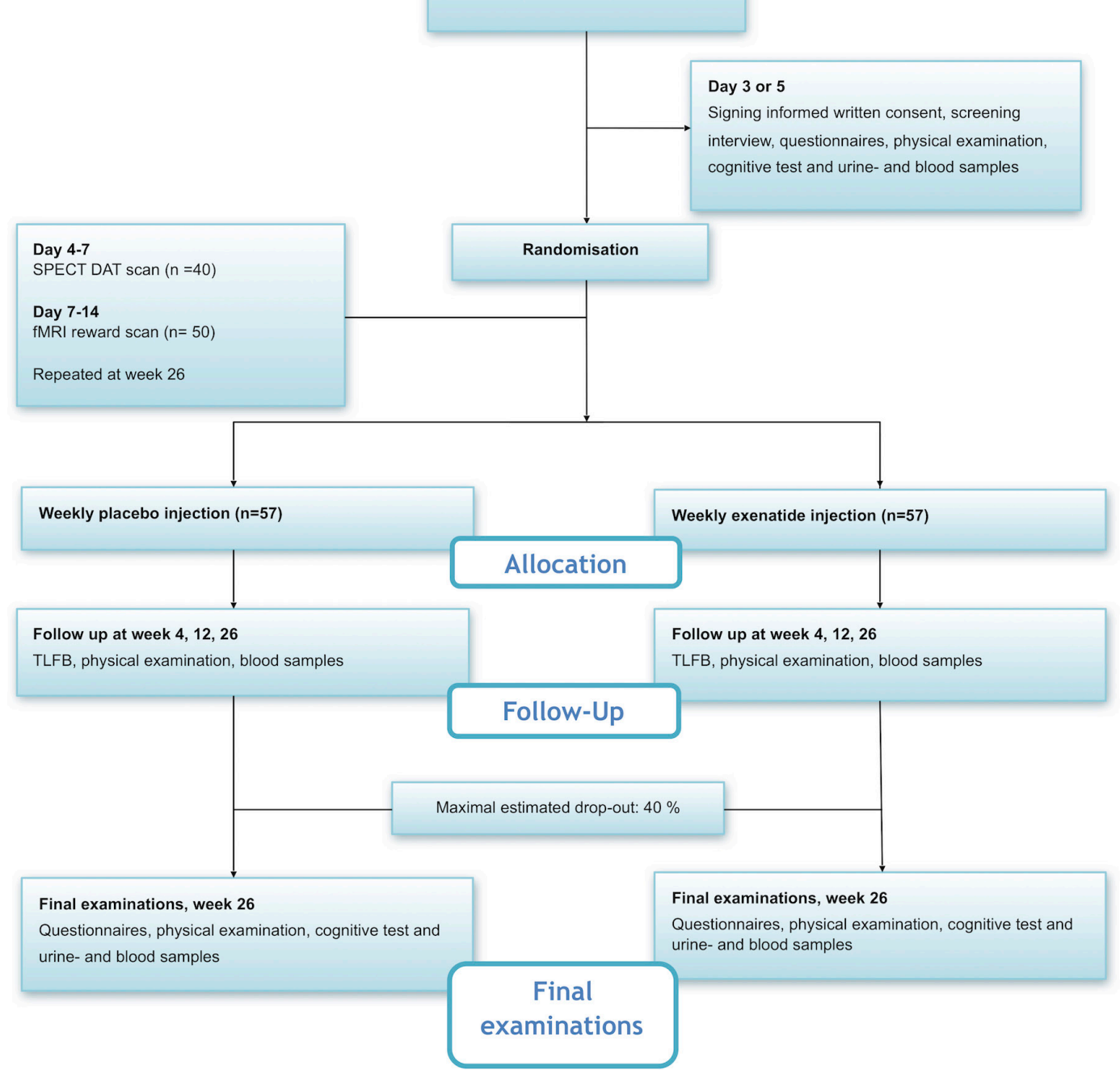

Figure 1 Study flow diagram. DAT, dopamine transporter; fMRI, functional MRI; SPECT, single-photon emission CT; TLFB, Timeline Followback.

- Concomitant pharmacotherapy with dopamine active drugs, such as some types of attention deficit hyperactivity disorder medication (methylphenidate).

- Receiving any investigational drug within the last 3 months.

- Use of weight-lowering pharmacotherapy within the preceding 3 months.

- Any other active substance use defined as a Drug Use Disorders Identification Test (DUDIT)-score ${ }^{31}>6$ (for men) $>2$ (for women) and fulfilling the criteria's for dependence of the substance according to the criteria of ICD-10 (except nicotine).

- Body mass index $<18.5 \mathrm{~kg} / \mathrm{m}^{2}$.

- Only for patients undergoing brain scans:

- Contraindications for MR scanning (magnetic implants, pacemaker, claustrophobia etc).
- Contraindications for SPECT scanning (radiation exposure, excluding background radiation but including diagnostic X-rays and other medical exposures exceeding $10 \mathrm{mSv}$ in the last 12 months, allergy towards iodine).

- Unable to speak and/or understand Danish.

- Any condition that the investigator feels would interfere with trial participation.

\section{Withdrawal criteria}

Patients are free to withdraw from the trial at any time without providing a reason therefore and without impact on further treatment at the Novavì outpatient clinics. The reason for withdrawal may be withdrawal of consent, treatment failure, adverse event, pregnancy discovered during the trial or profound increase in alcohol consumption. 
Failure to comply with clinical trial medication, that is, if the patient misses more than three consecutive injections or more than five injections in total leads to exclusion.

\section{Timeline Followback Method}

At weeks $0,4,12,20$ and 26, the examiner will-in close collaboration with the patient-fill out the TLFB schedule for the last 30 days, based on the weekly collected alcohol diaries. The TLFB has been extensively tested and evaluated $^{32}$ and has, in addition, been demonstrated to have a high test-retest reliability in previous studies. ${ }^{33}$ The information collected by the TLFB will be used to evaluate effects on the primary endpoint, that is, number of heavy drinking days.

\section{Blood analyses}

At every examination, a variety of routine blood samples will be drawn. This is to monitor that the patients have no serious adverse reactions to the treatment compromising liver, kidney or pancreatic function. HbAlc will only be analysed at week 0 and week 26 . At week 0 and week 26, blood will also be drawn for two advanced tests, proteomics and bone markers. At every examination, blood will be drawn for the advanced test PEth which is the biomarker with the best correlation to self-reported alcohol consumption and it can reflect alcohol consumption during several weeks prior to sampling. ${ }^{34}$ In the present study, we will be investigating the proteomic fingerprint as it is known that levels of humoral cytokines can be affected in alcohol-related liver diseases and that GLP-1RAs have an additional impact on humoral cytokines. ${ }^{35}$ We will also measure plasma levels of the bone markers collagen type $1 \mathrm{C}$-telopeptide and procollagen type $1 \mathrm{~N}$-terminal propeptide, as former studies show that another incretin hormone (GIP (Gastric Inhibitory Polypeptide)) reduces bone resorption. ${ }^{36}$

\section{Urine analyses}

At baseline (week 0) and at the final examination (week 26), a urine sample will be collected. This is for a routine screening of albumin:creatinine ratio and oxidative stress parameters. Oxidative stress, that is, excessive reactive oxygen species, can cause cell-damaging effects through oxidative modification of macromolecules leading to their inappropriate functions. Such oxidative modification is related to cancers, ageing, and neurodegenerative and cardiovascular diseases. ${ }^{37}$ Studies in rats have shown that the GLP-1RA liraglutide may have a direct beneficial effect on oxidative stress and diabetic nephropathy. ${ }^{38}$

\section{Questionnaires}

To assess potential psychopathology and drug use, a number of questionnaires will be administered at week 0 and at week 26 including: quality of life (Short Form Health Survey (SF-36)), psychopathology (Symptom Checklist (SCL-92)), depression symptoms (Major Depression Inventory (MDI)), alcohol consumption (AUDIT (Alcohol Use Disorders Identification Test)), craving (Penn Alcohol Craving Scale (PACS)), smoking
(Fagerström Test for Nicotine Dependence) and drug use (DUDIT (Drug Use Disorders Identification Test)).

\section{Screen for Cognitive Impairment in Psychiatry test}

The Screen for Cognitive Impairment in Psychiatry (SCIP) is a brief $(<20 \mathrm{~min})$ and feasible neuropsychological instrument for screening for cognitive dysfunction in patients with psychotic and affective disorders ${ }^{39}$ and in healthy controls. ${ }^{40}$ It will be administered at week 0,4 and 26 in three parallel equivalent forms to minimise learning effects.

\section{Single-photon emission CT}

A subgroup of 40 patients will have a SPECT scan performed at baseline and after 26 weeks of treatment. We will use the SPECT brain scan with the DAT ligand 123I-2-b-carbomethoxy-3b-(4-iodophenyl)-N-(3-fluoropropyl) nortropane ((123I)-FP-CIT (Ioflupane 123I), DaTSCAN) administrated as a bolus injection. The (123I)-FP-CIT binding potential is used to calculate an estimate of DAT availability in regions of interest. As no human data are available on the effects of GLP-1RA in DAT availability, we also propose to investigate DAT availability in healthy, non-alcohol-dependent subjects. The study on the healthy subjects is performed in order to investigate possible acute effects of exenatide on DAT availability in the human brain. Possible long-term effects of GLP-1R stimulation will be explored through scans at week 26 in the present study.

\section{Functional MRI}

The neuroanatomical underpinnings of the possible treatment effects will be investigated using fMRI at week 0 and 26. We will investigate brain activity during exposure to alcohol cues and during SWM performance. Furthermore, we will evaluate the effects of exenatide versus placebo on functional connectivity in the brain during resting state, on structural connectivity and brain morphology. A subgroup of 50 patients will have an fMRI scan performed at baseline and after 26 weeks of treatment. The patients will undergo two different tasks presented in block paradigms to maximise sensitivity for blood-oxygen-level dependent signal change. In the first task, the patients will be shown a series of alcohol-related and neutral pictures. Following each block, the patients rate the intensity of their alcohol craving on a Visual Analogue Scale. ${ }^{9}$ In the second task, the SWM will be assessed using an N-back version design. ${ }^{41}$ To obtain comparable data from healthy controls in the alcohol and SWM paradigms (fMRI), we will scan 25 healthy participants. The participants will have no history of alcohol dependence and will be matched to the patients with respect to gender and age.

\section{Sample size calculation and randomisation}

The primary outcome measure (total number of heavy drinking days) was used for the sample size calculation. Based on data from the study by Johnson $e t a l,{ }^{42}$ where the reduction in the percentage of total number of heavy 
drinking days was $60.3 \%$ in the intervention group and $32.7 \%$ in the control group, with an alpha of $5 \%$, a power of $90 \%$ and with an estimated SD of 34.5, the estimated sample size is of 68 patients ( 34 in each group). With an estimated dropout rate of $40 \%$, a total number of 114 patients (57 patients in each arm) are needed.

The patients will be randomised into two groups with 57 patients in each group using the randomisation module in Research Electronic Data Capture (REDCap). The randomisation will be stratified in terms of age (two levels), sex (two levels) and baseline alcohol consumption (ie, number of heavy drinking days measured by TLFB) (four levels). The block sizes will be randomised between 2 and 4 . The random allocation sequence will be generated by an extern statistician by use of the $\mathrm{R}$ statistical package blockrand and uploaded in REDCap in accordance with REDCap's user guide and reference manual. ${ }^{43}$

Patients, investigators, other care givers performing assessments and persons performing data analysis will remain blinded from the time of randomisation to time of database unlock. In order to maintain the blinding of the patients, an unblinded nurse will perform the randomisation and prepare the injections. If a patient develops, an adverse reaction that requires knowledge of the treatment the randomisation will be broken for only that particular patient.

\section{Patient and public involvement}

No patients were involved in development of the research question or in designing the study, and the burden of the intervention is not assessed by patients themselves.

When signing the informed consent, patients are encouraged to fill in their email address, so they can receive the results of the study.

\section{Statistical analysis}

Before dividing participants into two groups, the statistical analysis plan will be uploaded at ClinicalTrials.gov. Analyses will be made by use of R software, ${ }^{44}$ with alpha set at 0.05 and two-sided testing. All analyses will be performed using the intention-to-treat principle on subjects, who were randomised and received at least one dose of the trial compound (exenatide or placebo). Missing data will be imputed using multiple imputations, and a sensitivity analysis will be undertaken to evaluate and compare imputation results to complete case analyses. Multiple linear regression and logistic regression analyses will be used for the analyses, and data will be controlled for possible confounders, for example, baseline alcohol consumption, social status, age etc in addition to the treatment.

\section{Endpoints}

The primary endpoint is per cent reduction in total number of heavy drinking days, defined as days with an excess intake of $60 / 48 \mathrm{~g}$ of alcohol per day (men and women, respectively) the previous 30 days from baseline to follow-up after 26 weeks of treatment, measured by TFLB method.
The secondary endpoints include changes in total alcohol consumption ( $\mathrm{g} / 30$ days measured by TLFB), changes in number of days without alcohol consumption and PACS score, change in AUDIT score, change in DUDIT score, change in cognitive performance on the SCIP-test, change in the liver parameters gamma-glutamyltransferase, alanine aminotransferase and PEth. Other parameters will be mean cell volume (MCV), changes in body weight, blood pressure, pulse, overall glycaemic control parameters (HbA1c), kidney function (p-creatinine, eGFR and urine albumin/creatinine ratio) and measures of health (SF-36 and SCL-92).

In addition to these clinical outcome parameters, we will explore the possible neuromolecular effects by measuring striatal DAT availability before and after administration of exenatide by use of SPECT. The possible neuroanatomical underpinnings of exenatide will be investigated by use of fMRI. Both examinations will be performed in two subgroups of patients treated with either exenatide or placebo. To have comparable standard data in this fMRI alcohol-related paradigm, we will include 25 healthy participants with no record of alcohol dependence.

The Consolidated Standards of Reporting Trials guidelines will be followed when final study data are reported.

\section{Ethical considerations}

On ClinicalTrials.gov, it can be identified by the ID NCT03232112. Please see online supplementary appendix A for further details. The protocol has version control and dates as identifiers. Any amendments have to be approved by the above-mentioned authorities before implementation.

\section{DISCUSSION}

Data from animal studies suggest that the inhibitory effects of the GLP-1RA exendin-4 reduce alcohol consumption in rodents and this effect is likely mediated by stimulation of the DAT ${ }^{26}$ ). So far no human studies have been performed and the present trial therefore serves to investigate the effects of the GLP-1RA exenatide on alcohol consumption in patients with alcohol dependence as well as the associated neurobiological mechanisms. This trial is the first RCT (Randomized Clinical Trial) to investigate the effects of GLP-1R stimulation on alcohol consumption in patients diagnosed with alcohol dependence.

\section{Limitations}

The measurement of the primary endpoint of the study, that is, change in heavy drinking days, is self-reported and retrospective, and might therefore have limited reliability. In the present study, we use the TLFB method which has been extensively tested and evaluated.$^{32}$ Self-reported measurements can be influenced by several factors including social factors characteristics in the respondent group. ${ }^{45}$ For example, it is known that patients with alcohol dependence tend to describe themselves more negatively, that is, having more heavy drinking days etc, than suggested by data from more objective sources, for 
example, blood samples. ${ }^{32}$ However, when patients have alcohol in the blood, the opposite is seen, that is, an underestimation of the alcohol intake. ${ }^{32}$ Thus, to limit the possible bias from different factors, the TLFB will be filled out in close cooperation with the patient in a standardised setting. In addition, the patients will do a breath alcohol test prior to all examinations.

Another limitation of the study is that the treatment is not evaluated long term. We also considered adding a third arm comparing exenatide to one of the established add-on treatments (all pharmacological treatments is considered as an add-on to CBT). However, adding a third arm would have increased the complexity and cost of the trial considerably. A weakness of the present study is the lack of blinded placebo pens making weekly injections of exenatide by the study nurse necessary, which increases the risk of selection bias, as the design requires a very compliant patient, that is, patients having less resources might not participate. Additionally, some patients might choose not to participate because of needle phobia.

\section{Strengths}

A significant advantage of the present study is the extensive use of unbiased, biological measurements, that is, biomarkers in blood and urine and brain scans. A systematic review of the biomarker PEth thus showed a significant statistical difference when comparing heavy drinkers (ie, $>60 \mathrm{~g}$ of alcohol per day) from persons consuming less, ${ }^{46}$ making it very useful in the present study as we will be able to assess the correlation between the self-reported alcohol intake and PEth. Another advantage is the use of the brain imagining techniques SPECT and fMRI. The brain scans will allow the investigation of the possible neuroanatomical underpinnings of the treatment. A definite strength of the study is the long treatment period, that is, 26weeks, when comparing to similar studies with study durations of typically 8-12weeks. ${ }^{48}$ This relatively long treatment period will allow a better understanding of the true effects of exenatide as it corresponds to a more realistic setting with an ongoing risk of relapse persisting way longer than just a few months. ${ }^{4}$ Also, the design, that is, double-blinded, randomised, placebo-controlled, is an advantage as it reduces experimental bias, ensures balance in the two treatment groups and gives a direct estimate of the possible effect of exenatide. In addition, the present injection set-up allows us to verify, that the injections have been administered to the patients.

\section{Perspectives}

If GLP-1R stimulation proves efficacious in the treatment of alcohol dependence, it can be implemented in future treatment relatively easy as exenatide is already used in the clinic and the injections are designed for self-distribution. Further, per oral GLP-1RAs may be on the market within a few years, which would possible increase compliance even more. In addition, assessment of the neuronal underpinnings of the potential treatment effects will increase insight into neurobiological targets for future treatments.

\section{Trial status}

Patient enrolment started in August 2017 and is ongoing until 114 patients have been randomised and received first injection.

\section{Author affiliations}

${ }^{1}$ Psychiatric Centre Copenhagen, University Hospital of Copenhagen, Copenhagen, Denmark

${ }^{2}$ Department of Psychology, University of Copenhagen, Copenhagen, Denmark ${ }^{3}$ Neurobiology Research Unit, Copenhagen University Hospital and Center for Integrated Molecular Brain Imaging, Copenhagen, Denmark

${ }^{4}$ The Novavi Outpatient Clinics Copenhagen, Copenhagen, Denmark

${ }^{5}$ Department of Addictive Behaviour and Addiction Medicine, Central Institute of Mental Health, Medical Faculty Mannheim/Heidelberg University, Mannheim/ Heidelberg, Germany

${ }^{6}$ Department of Anesthesiology, Yale University, New Haven, Connecticut, USA

${ }^{7}$ National Institute on Drug Abuse, National Institutes of Health, Bethesda, Maryland, USA

${ }^{8}$ National Institute of Public Health, University of Southern Denmark, Copenhagen, Denmark

${ }^{9}$ Gastrounit, Medical Division, Copenhagen University Hospital Hvidovre, Hvidovre, Denmark

${ }^{10}$ Department of Public Health, Section of Biostatistics, University of Copenhagen, Copenhagen, Denmark

${ }^{11}$ Department of Clinical Medicine, Faculty of Health and Medical Sciences,

University of Copenhagen, Copenhagen, Denmark

${ }^{12}$ Steno Diabetes Center Copenhagen, University of Copenhagen, Gentofte, Denmark

Acknowledgements The authors thank the staff, especially the project nurses at the Novavi alcohol outpatient clinics for their support and help with the patient population including Bydureon/placebo injections, as well as statistician Majken Sey for statistical support.

Contributors According to the definition given by the International Committee of Medical Journal Editors (ICMJE), all the authors qualify for authorship. AF-J and TV made the first draft of the study protocol. KKA, MKK, ASB, NID, MEJ, KWM, HR, TPF, HB, NV, GMK and UB have made substantial contributions to the study design. KKA, MKK, CE and AF-J undertook the statistical power calculations. KWM, PMF, MKK, GKT and GMK undertook the final design of the fMRI experiment. SV-K designed the ALCUE fMRI-paradigm. KKA, GKT and GMK undertook the final design of the SPECT experiment. KKA wrote the first draft of the manuscript based on the study protocol. All authors contributed with critical revision of the manuscript for important intellectual content and have approved the final manuscript.

Funding This study is supported by the Research Foundation (Mental Health Services, Capitol Region of Denmark), The Research Foundation (Capitol Region of Denmark), The Ivan Nielsen Foundation, the A.P. Møller and wife Chastine Mc-Kinney Møllers Family Foundation, the Lundbeck Foundation and the Novavi Foundation.

Competing interests TV has received lecture fees from Amgen, Astra Zeneca, Boehringer Ingelheim Pharmaceuticals, Bristol-Myers Squibb, Eli Lilly and Company, Merck Sharp \& Dohme, Novo Nordisk, Sanofi and Zealand Pharma, and is a member of the Advisory Boards of Astra Zeneca, Boehringer Ingelheim Pharmaceuticals, Bristol-Myers Squibb, Eli Lilly and Company, Merck, Sharp \& Dohme, Novo Nordisk and Sanofi. AF-J has received an unrestricted grant from Novo Nordisk A/S for another project.

Patient consent Not required.

Ethics approval The study is approved by The Regional Committee on Biomedical Research Ethics (journal number H-17003043), The Danish Data Protection Agency (protocol number RHP-2017-029) and the Danish Medical Agency (EudraCT 2016--003343--11).

Provenance and peer review Not commissioned; externally peer reviewed.

Open access This is an open access article distributed in accordance with the Creative Commons Attribution Non Commercial (CC BY-NC 4.0) license, which permits others to distribute, remix, adapt, build upon this work non-commercially, and license their derivative works on different terms, provided the original work is properly cited and the use is non-commercial. See: http://creativecommons.org/ licenses/by-nc/4.0/ 
(c) Article author(s) (or their employer(s) unless otherwise stated in the text of the article) 2018. All rights reserved. No commercial use is permitted unless otherwise expressly granted.

\section{REFERENCES}

1. Wittchen HU, Jacobi F, Rehm J, et al. The size and burden of mental disorders and other disorders of the brain in Europe 2010. Eur Neuropsychopharmacol 2011;21:655-79.

2. Anton RF, O'Malley SS, Ciraulo DA, et al. Combined pharmacotherapies and behavioral interventions for alcohol dependence: the COMBINE study: a randomized controlled trial. JAMA 2006;295:2003-17.

3. Schuckit MA. Alcohol-use disorders. Lancet 2009;373:492-501.

4. Kohn R, Saxena S, Levav I, et al. The treatment gap in mental health care. Bull World Health Organ 2004;82:858-66.

5. Sundhedsstyrelsen. nye udmelding vedrørende alkohol. Natl Board Heal. 2010:1-6. http://www.sst.dk/ /media/Sundhed og forebyggelse/Alkohol/AlkoholudmeldingAug2010/NOTAT_ alkoholudmelding_aug_2010.ashx

6. Sundhedsstyrelsen. Alkoholstatistik 2015. 9, 2015.

7. Hansen AB, Hvidtfeldt UA, Grønbæk M, et al. The number of persons with alcohol problems in the Danish population. Scand J Public Health 2011;39:128-36.

8. Morgenstern J, Longabaugh R. Cognitive-behavioral treatment for alcohol dependence: a review of evidence for its hypothesized mechanisms of action. Addiction 2000;95:1475-90.

9. Vollstädt-Klein S, Loeber S, Kirsch M, et al. Effects of cue-exposure treatment on neural cue reactivity in alcohol dependence: a randomized trial. Biol Psychiatry 2011;69:1060-6.

10. Vollstädt-Klein S, Hermann D, Rabinstein J, et al. Increased activation of the ACC during a spatial working memory task in alcohol-dependence versus heavy social drinking. Alcohol Clin Exp Res 2010;34:771-6.

11. Sundhedsstyrelsen. Behandling af alkoholafhængighed. National klinisk retningslinje for behandling af alkoholafhængighed. https:// sundhedsstyrelsen.dk/da/nyheder/2015/ /media/DA9C87FC4B3F 490E8C480B5E692F125E.ashx (accessed 17 Feb 2017).

12. MARTENSEN-LARSEN O. Treatment of alcoholism with a sensitizing drug. Lancet 1948;2:1004.

13. Snyder JL, Bowers TG. The efficacy of acamprosate and naltrexone in the treatment of alcohol dependence: a relative benefits analysis of randomized controlled trials. Am J Drug Alcohol Abuse 2008;34:449-61.

14. Blodgett JC, Del Re AC, Maisel NC, et al. A meta-analysis of topiramate's effects for individuals with alcohol use disorders. Alcohol Clin Exp Res 2014;38:1481-8.

15. Mann K, Bladström A, Torup L, et al. Extending the treatment options in alcohol dependence: a randomized controlled study of as-needed nalmefene. Biol Psychiatry 2013;73:706-13.

16. Holst JJ. The physiology of glucagon-like peptide 1. Physiol Rev 2007;87:1409-39.

17. Vilsbøll T, Krarup T, Madsbad S, et al. Both GLP-1 and GIP are insulinotropic at basal and postprandial glucose levels and contribute nearly equally to the incretin effect of a meal in healthy subjects. Regul Pept 2003;114(2-3):115-21.

18. Astrup A, Rössner S, Van Gaal L, et al. Effects of liraglutide in the treatment of obesity: a randomised, double-blind, placebo-controlled study. Lancet 2009;374:1606-16.

19. Runge S, Schimmer S, Oschmann J, et al. Differential structural properties of GLP-1 and exendin-4 determine their relative affinity for the GLP-1 receptor N-Terminal extracellular domain ${ }^{\dagger}$. Biochemistry 2007;46:5830-40.

20. Dickson SL, Shirazi RH, Hansson C, et al. The glucagon-like peptide 1 (GLP-1) analogue, exendin-4, decreases the rewarding value of food: a new role for mesolimbic GLP-1 receptors. J Neurosci 2012;32:4812-20.

21. Egecioglu E, Steensland P, Fredriksson I, et al. The glucagonlike peptide 1 analogue Exendin-4 attenuates alcohol mediated behaviors in rodents. Psychoneuroendocrinology 2013;38:1259-70.

22. Shirazi RH, Dickson SL, Skibicka KP. Gut peptide GLP-1 and its analogue, Exendin-4, decrease alcohol intake and reward. PLoS One 2013;8:e61965-7.
23. Davis JF, Schurdak JD, Magrisso IJ, et al. Gastric bypass surgery attenuates ethanol consumption in ethanol-preferring rats. Biol Psychiatry 2012;72:354-60.

24. Nutt DJ, Lingford-Hughes A, Erritzoe D, et al. The dopamine theory of addiction: 40 years of highs and lows. Nat Rev Neurosci 2015;16:305-12.

25. Skibicka KP. The central GLP-1: implications for food and drug reward. Front Neurosci 2013;7:1.

26. Reddy IA, Pino JA, Weikop P, et al. Glucagon-like peptide 1 receptor activation regulates cocaine actions and dopamine homeostasis in the lateral septum by decreasing arachidonic acid levels. Trans/ Psychiatry 2016;6:e809.

27. Fink-Jensen A, Vilsbøll T. Glucagon-like peptide-1 (GLP-1) analogues: A potential new treatment for alcohol use disorder? Nord J Psychiatry 2016;70:561-2.

28. Babor TF, Higgins-biddle JC, Saunders JB, et al; The Alcohol Use Disorders Identification Test.

29. Atmp F, Studies NS, Trials HC, et al. Recommendations related to contraception and pregnancy testing in clinical trials. 2, 2009.

30. Ludema KC. European public assessment reports 2016:1-64.

31. Berman AH, Bergman $\mathrm{H}$, Palmstierna T, et al. Evaluation of the Drug Use Disorders Identification Test (DUDIT) in criminal justice and detoxification settings and in a Swedish population sample. Eur Addict Res 2005;11:22-31.

32. Sobell LC, Sobell MB. Alcohol Consumption Measures, 1995.

33. Sobell MB, Sobell LC, Klajner F, et al. The reliability of a timeline method for assessing normal drinker college students' recent drinking history: utility for alcohol research. Addict Behav 1986;11:149-61.

34. Walther L, de Bejczy A, Löf E, et al. Phosphatidylethanol is superior to carbohydrate-deficient transferrin and $\gamma$-glutamyltransferase as an alcohol marker and is a reliable estimate of alcohol consumption level. Alcohol Clin Exp Res 2015;39:2200-8.

35. Neuman MG. Cytokines-central factors in alcoholic liver disease. Alcohol Res Health 2003;27:307-16.

36. Christensen MB, Lund A, Calanna S, et al. Glucose-Dependent Insulinotropic Polypeptide (GIP) Inhibits Bone Resorption Independently of Insulin and Glycemia. J Clin Endocrinol Metab 2018;103:288-94.

37. Wang JX, Gao J, Ding SL, et al. Oxidative Modification of miR-184 Enables It to Target Bcl-xL and Bcl-w. Mol Cell 2015;59:50-61.

38. Hendarto $H$, Inoguchi $T$, Maeda $Y$, et al. GLP-1 analog liraglutide protects against oxidative stress and albuminuria in streptozotocininduced diabetic rats via protein kinase A-mediated inhibition of renal NAD(P)H oxidases. Metabolism 2012;61:1422-34.

39. Demant KM, Almer GM, Vinberg M, et al. Effects of cognitive remediation on cognitive dysfunction in partially or fully remitted patients with bipolar disorder: study protocol for a randomized controlled trial. Trials 2013:14:378.

40. Ott CV, Bjertrup AJ, Jensen JH, et al. Screening for cognitive dysfunction in unipolar depression: Validation and evaluation of objective and subjective tools. J Affect Disord 2016;190:607-15.

41. Miskowiak K, Inkster B, O'Sullivan U, et al. Differential effects of erythropoietin on neural and cognitive measures of executive function 3 and 7 days post-administration. Exp Brain Res 2008;184:313-21.

42. Johnson BA, Ait-Daoud N, Bowden CL, et al. Oral topiramate for treatment of alcohol dependence: a randomised controlled trial. Lancet 2003;361:1677-85.

43. Randomization Module. 2015 http://cri.uchicago.edu/wp-content/ uploads/2015/12/REDCap-Randomization-Module.pdf.

44. R Development Core Team. A language and environment for statistical computing: The R Foun(the R Foundation for Statistical Computing.), 2011

45. Del Boca FK, Darkes J. The validity of self-reports of alcohol consumption: state of the science and challenges for research Addiction 2003;98 Suppl 2:1-12.

46. Viel G, Boscolo-Berto R, Cecchetto G, et al. Phosphatidylethanol in Blood as a Marker of Chronic Alcohol Use: A Systematic Review and Meta-Analysis. Int J Mol Sci 2012;13:14788-812.

47. Kranzler HR. Pharmacotherapy of alcoholism: gaps in knowledge and opportunities for research. Alcohol Alcohol 2000;35:537-47.

48. Mann K. Pharmacotherapy of alcohol dependence. CNS Drugs 2004;18:485-504. 